\title{
Biodiversity of chloramphenicol-resistant mesophilic heterotrophs from Southeast Asian aquaculture environments
}

\begin{abstract}
In the present study, samples of pond water, sediment and farmed species were collected at 12 fish and shrimp farms in Malaysia, Thailand and Vietnam to determine the biodiversity and environmental distribution of chloramphenicol-resistant $(\mathrm{CmR})$ mesophilic heterotrophs in Southeast Asian aquaculture sites. Following isolation on Iso-Sensitest agar supplemented with $35 \mu \mathrm{g} \mathrm{ml}-1 \mathrm{Cm}$ and dereplication using (GTG)5-PCR fingerprinting, 557 genotypically unique $\mathrm{CmR}$ strains were subjected to polyphasic identification. The 557 mesophilic heterotrophic $\mathrm{CmR}$ isolates represented 18 different genera largely dominated by the genera Escherichia (40.2\%), Pseudomonas (11.7\%), Acinetobacter (11.1\%), Klebsiella (7.5\%) and Bacillus (5.9\%). A total of $439 \mathrm{CmR}$ isolates were further assigned to 31 described species or species groups, mainly including organisms that have been associated with various human opportunistic infections such as Escherichia coli $(n=219)$, Pseudomonas putida $(n=47)$, Klebsiella pneumoniae $(n=38)$ and Acinetobacter baumannii $(n=23)$. Strains of Escherichia, and most notably, of E. coli, were the only common group of $\mathrm{CmR}$ heterotrophs irrespective of country, sample type or farm type. Together with other predominant but less widespread groups such as acinetobacters and pseudomonads, the results of this biodiversity study suggest that E. coli can be regarded as a potential indicator of $\mathrm{Cm}$ resistance in Southeast Asian aquaculture environments.
\end{abstract}

Keyword: Taxonomy, Antibiotic resistance, Human opportunists 